\title{
Reduction of Product Portfolio Complexity Based on Process Analysis
}

\author{
Aleksandra STASKIEWICZ ${ }^{a, 1}$, Lars HVAM $^{\mathrm{a}}$ and Anders HAUG ${ }^{b}$ \\ a Technical University of Denmark \\ ${ }^{\mathrm{b}}$ University of Southern Denmark
}

\begin{abstract}
The increased demand for product variety has implied that many manufacturing companies are struggling with managing product complexity. This article suggests a framework for combined modeling of product variants and the process flow in production and assembly for customized products. The aim of the framework is to create a visual model that illustrates the product variety relative to the process flow and provides transparency of product variety in the different process steps. Literature has suggested various methods and techniques. These provide means for reducing complexity based on analysis of the end product, but do not pay much attention to understanding where in the production and assembly processes, this variance occurs. The suggested models form a basis for analyzing and reducing product complexity based on a visual model of the product variety in each process step. The models gave rise to a reduction of the SKUs with $33 \%$ without losing product variety offered to the end customer. The initial test of the framework and models in the case company showed that the models can provide new insight into the product variety, which forms a solid basis for making decisions on reducing product portfolio variety and adjusting the order decoupling point.
\end{abstract}

Keywords. Internal product portfolio complexity, product rationalization, productprocess complexity, product complexity mapping

\section{Introduction}

Industry companies tend to increase product variety with the belief that this will increase their competitive advantage and their market share [1]. On the other hand, this increase in product variety is driven by customers' demand for unique applications [2]. Due to this increased product variety, many businesses face raising levels of complexity in their product architectures, product portfolios, and internal processes [3]. In previous studies, the majority of executives express concerns that these rising levels of complexity negatively impact operational performance on e.g., quality, delivery performance, and profitability $[4,5]$. Since operational performance and production strategy are the keys to competitiveness and overall business performance [6], it is of great importance for companies to understand the impact of increased product variety on operational performance.

To cope with the increasing levels of complexity in the product portfolio, many companies try to limit product variety by using a comprehensive complexity reduction approach, which targets final products offered to customers for rationalization - i.e., Stock Keeping Unit (SKU) reduction [7]. However, case studies show that managers

\footnotetext{
${ }^{1}$ Corresponding Author. amst@dtu.dk
} 
often struggle with product rationalization decisions due to limited understanding of the relationship between product variety and raising complexity levels in product architecture, product portfolio and internal processes [2, 3]. Several researchers have suggested that the reason for such problems is a lack of understanding of the concept of product portfolio architecture complexity (PPAC) and its' effect on operational performance [8]. However, research into diversification, product platforms, and similar PPAC-related topics has led to inconsistent guidance and contradictory findings of how to offer adequate product variety to customers and avoid introducing increasing levels of complexity in product architectures and product portfolios [9, 10,11].

Existing research focusing on how to reduce increased product variety has not paid much attention to how the increasing levels of complexity in product architectures and product portfolios are linked to the internal processes of companies. In some cases, however, it seems to be crucial to understand this link. To address this gap, this paper proposes process-focused approach for addressing product complexity issues. The relevance and usefulness of the framework are demonstrated by a case study of a company where it was applied.

The remainder of the paper is structured as follows. First, relevant literature is discussed, on which basis an approach for addressing product complexity is proposed. Next, the research method is described, after which the findings from the case study are presented. The paper ends with a discussion of the findings and conclusions being drawn.

\section{Literature}

To provide a basis for the creation of a process-focused framework for the reduction of product complexity, this literature review discusses the topics of SKU and product complexity management.

In production and operations management, companies often have to deal with many different products, or Stock Keeping Units (SKUs). Here, SKUs refer to items of stock that are completely specific as to function, style, size, color, and, usually, location [12, p. 32]. The production and inventory policies of these different SKUs are influenced by the characteristics of the product. Differences in annual sales volume, predictability of demand, product value, or storage requirements might result in different production and inventory policies. As a consequence, companies that sell a wide variety of SKUs often struggle with controlling their production and inventory systems. Therefore, in real-life situations, it is generally seen as advantageous to distinguish a limited number of SKU classes based on the characteristics of these SKUs. This enables companies to make decisions on production, inventory management, and customer service strategies for entire SKU classes rather than for each product separately.

Often product complexity is evaluated through traditional SKU tail-cutting Pareto analysis, where the slow-moving, low volume product variants are eliminated from the product portfolio [16]. Many practitioners highlight two main disadvantages with this SKU reduction approach [16]. Firstly, it tends to be one-time event. Secondly, it does not address the supply chain complexity and ignores operational performance. This is because the SKU reduction approach targets products measured by revenues rather than costs. [13]. In the end, the sales improvements are more likely to offset the compromised operational performance, and the product complexity will come back with a new product introduction. 
SKUs refer to items of stock that are completely specific as to characteristics, such as function, style, size, color, location, and similar. SKU characteristics mainly result from customer demand and the characteristics of the product. The number of SKU classes and the boundaries between them are essentially management decisions [14]. In existing literature, different approaches have been presented on SKU classification, but little foundation/guideline is offered for individual choices. When deciding on the number of SKU classes and class boundaries, we should aim for a trade-off between performance and complexity. While the best performance could theoretically be expected to be achieved by creating different classes for each product, this will come at the expense of complexity [14]. On the other hand, using only one class will result in relatively poor performance [14].

The main aim of any SKU classification is to use the similarity of products with regards to different properties to systematically classify products. In this context, Van Kampen et. al. [14] identify four main categories for characteristics in SKU classification: volume, product, customer, and timing. Additionally, Krishnan and Ulrich [15] distinguish four perspectives on product properties: marketing, organizations, engineering design, operations management. In this paper, we focus on the classification of products from the production and operations management perspective.

As mentioned in the Introduction, it has been suggested that there is lack of understanding of the concept of product portfolio architecture complexity (PPAC) and its' effect on operational performance [8], which is reflected inconsistent guidance and contradictory findings $[9,10,11]$. One of the exceptions is Jacob and Swink [11], who study the impact of product portfolio architecture complexity (PPAC) described by multiplicity, diversity, and interrelatedness on operational performance. However, in their research, they do not discuss how to reduce complexity by providing transparency in PPAC and how the PPAC is linked to process structure. Therefore, it may be difficult to apply their approach when doing product rationalization. Another example is Van Kampen et.al [14], who provide a framework on how SKU classification techniques can be used to support operations management and production strategy decisions by managers. However, in their study, they did not asses how this SKU classification techniques could be used to assess business performance, how it should be linked to internal processes, and how it should be used to reduce complexity levels in PPAC.

The concept of trade-offs is a key discussion of product variety management. The trade-off of increased product variety is the need to balance the increased revenue gained from greater variety with the increasing unit costs gained from producing or stocking lower variety [10]. However, there is a little understanding of how product variety impacts different production system designs, and how to counter this impact [1].

The literature on SKU reduction discusses methods for classification and reduction of SKUs, but only very little focus is on how to relate SKU reduction to the process flow. The literature on complexity management discusses the relation between product and process complexity, however, only limited research can be found on how to model and visualize this relation.

\section{A framework for reduction of SKU complexity}

Based on the literature review, this section describes a framework for the reduction of SKU complexity. The suggested process can support product structure mapping to reduce complexity at different levels in the product structure and link to the supply chain. 
The process is organized under the two overall phases "AS-IS analysis" and "TOBE design", under which a set of process steps are defined. The framework is shown in Table 1 and subsequently explained. It should be noted that the suggested framework focuses only on the analytical part of the project, as opposed to the implementation of initiatives.

Table 1. A framework for SKU reduction

\begin{tabular}{ccc}
\hline Framework & Steps & Action \\
\hline AS-IS analysis & $1 \mathrm{a}$ & Define product and process scopes of the project \\
& $1 \mathrm{~b}$ & Identify and organize SKU characteristics \\
& $1 \mathrm{c}$ & Map product-process complexity \\
& $1 \mathrm{~d}$ & Identify complexity performance trade-offs \\
TO-BE scenario & $2 \mathrm{a}$ & Identify possible SKU reduction initiatives \\
& $2 \mathrm{~b}$ & Choose SKU reduction initiative \\
\hline
\end{tabular}

\subsection{Phase 1: AS-IS analysis}

\section{Step 1a: Define product and process scope}

The first step is to delimit the analysis to the product families and parts of the process flow with the best chances for producing benefits. Figure 1 explains that the focus of traditional product complexity analysis is only at the end products, and that in this framework, the focus is on manufacturing and assembly process. For this reason, to delimit the work and data needed for conducting analysis, the study is scoped based on selecting a limited number of products and process steps. The product families to study are identified by analyzing product variety, costs, delivery performance, quality, and other performance data from production and assembly for each product family. Product families for which there are observed low performance in terms of some of these performance parameters would be candidates for a study of the complexity at different process steps. The process steps are selected based on the same considerations, so that the analysis will include the process steps, where the product variety is expected to have an impact on the performance of the processes.

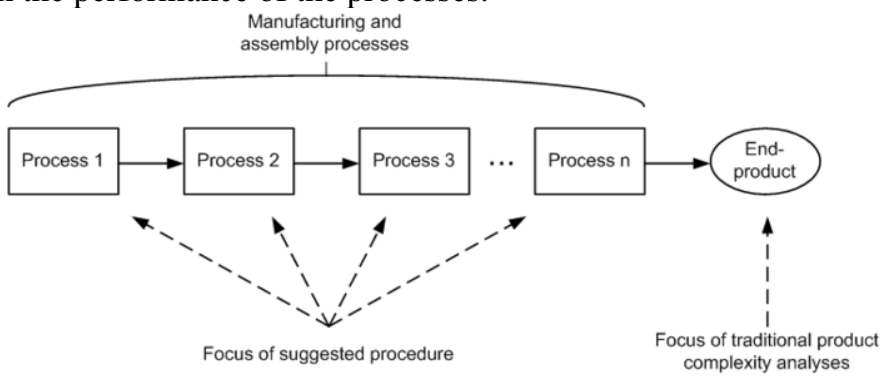

Figure 1. Focus of the suggested framework.

To identify the first candidates for this analysis, interviews or surveys with employees such as production, warehouse, logistic managers, and product developers should be conducted. This is to map the process steps in the supply chain at which product architecture and product portfolio drives complexity. The data gathering includes information about which product families drive complexity product portfolio and product architectures, mapping process steps in supply chains, and the impact of the product families on different process steps.

The numerical analysis to identify which product families drives complexity in the product portfolio is the tail-cutting Pareto analysis [16] and internal KPIs study on performance measures [16]. These analyses should be conducted to identify low- 
performing products and significantly decrease or disproportion in performance measure between different product families. The data that can be used for the given analysis include financial and operational performance data from enterprise resource planning (ERP) system. The information about KPIs on performance measures should be also possible to be provided by indicated stakeholders.

Furthermore, the initial candidates should be analyzed in terms of their SKU proliferation at different process steps in the supply chain and to identify if this can have the potential cause for decrease in performance measures. The data that can be used for given analysis include product master data like product design and bill of material information from product lifecycle management (PLM) systems. The indicated data can be helpful in quantifying levels of multiplicity, diversity, and interrelatedness. To conduct this analysis, data should be structured and organized in the following way. First, the product information for each SKU should be collected, as well as a corresponding performance measure by which we want to justify our product variety, e.g., product volume, product sales, etc. Furthermore, from the PLM systems, information such as the number of product variants, percentage of common components, dates of design, and product launches should be collected as they will be direct indicators for SKU classes.

\section{Step 1b: Identify and organize SKU characteristics}

SKU refers to items of stock that are specific to their combinations of characteristics, such as function, style, size, color, and stock location. In this framework, it is suggested to distinguish the SKU characteristics resulting from the product features and the SKU characteristics resulting from customer demand. This split to product SKU characteristics and customer SKU characteristics will provide the information on SKU classification the number of SKU classes and class boundaries between these two characteristics types. Hereby, it is possible to examine the trade-off between performance and increasing levels of complexity in the product architecture, product portfolio, and process in the process steps in the supply chain.

\section{Step 1c: Map product-process complexity}

The next step is to map the identified SKU classes and inherited product and customer SKU characteristics and link them to the process steps. The SKU classes and SKU characteristics should be mapped using variant trees methods. This will show how the complexity levels arise in the product architectures and product portfolio. Figure 2 provides an example of how the product SKU characteristics can be classified and furthermore how the customer SKU characteristics make these SKU classes the SKU used for product variety offered to the market. The mapping of process steps is done based on lists of operations for defined product family. The selection of steps included in this mapping should be related to the identified SKU characteristics. As an example, if a product is available with four different battery sizes, we need to select this process step in the process flow where this battery size will matter for the increase in product variants. The number of product variants in each process step is found based on the product data in, for example, PLM systems, and it can also be found in the lists of operations for a product family. 


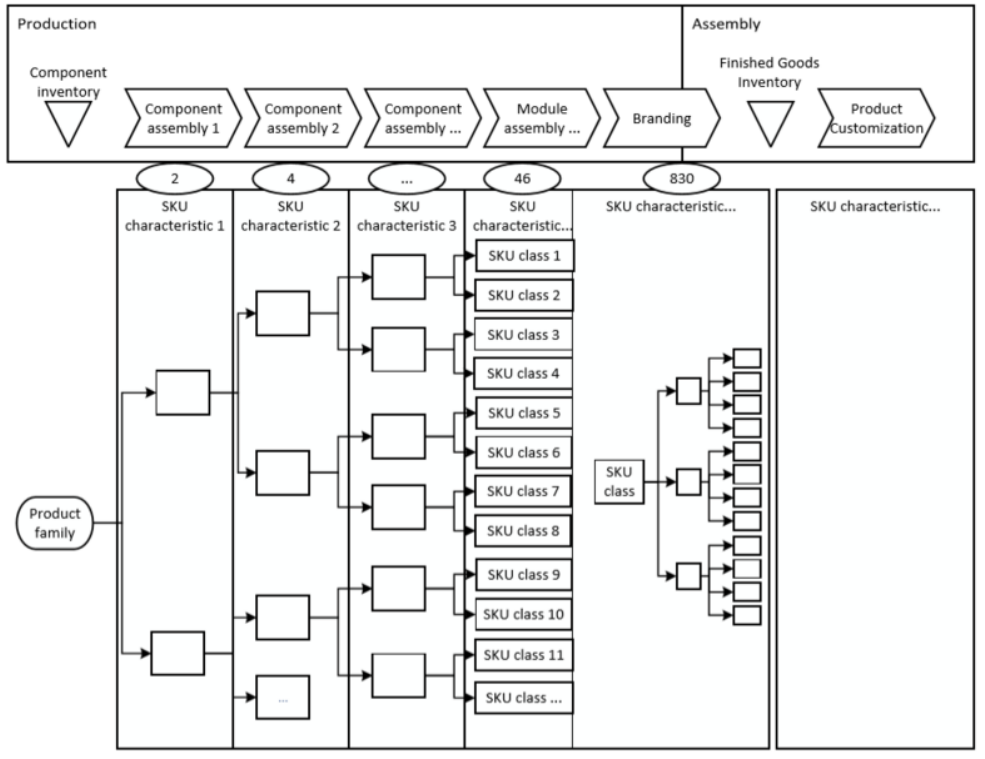

Figure 2. Mapping of product-process complexity framework based on company case.

\section{Step 1d: Identify complexity-performance trade-offs}

The next step is to analyze trade-offs between performance measures and increasing levels of complexity by using relevant classification techniques. Various classification approaches and techniques exist depending on what goal is to be achieved. One of the well-known classification approaches is ABC analysis [12], which usually classifies product groups based on either demand value or demand volume. Another well-known approach is the FNS (Fast, Normal, Slow) technique [20], which distinguishes product classes based on demand rate. It is important to realize that data on demand value, demand volume, demand rate, etc. can be found only for the product variants. For this reason, once the classification technique will be conducted, the results obtained for product variants should be properly assigned to the SKU classes.

Figure 3 provides an example of how the results SKU classification can be assigned to the SKU classes. In this example performance is measured using traditional Pareto analysis based on demand volumes, where products in category A are the product variants that contribute to $80 \%$ of demand volume, products $\mathrm{B}$ are the product variants that contribute to next $15 \%$ of demand volume, and product $\mathrm{C}$ are the product variants that contribute to last $5 \%$ of demand volume. The performance of a product at different levels is calculated using a weighted average, where value is the cumulated contribution to demand volume and the weighted factor is the demand volume. This can determine the performance of our product variety at different levels. However, while the best performance could theoretically be expected to be achieved by creating different classes for each product, this will come at the expense of increasing levels of complexity. On the other hand, using only one class will result in relatively poor performance. 


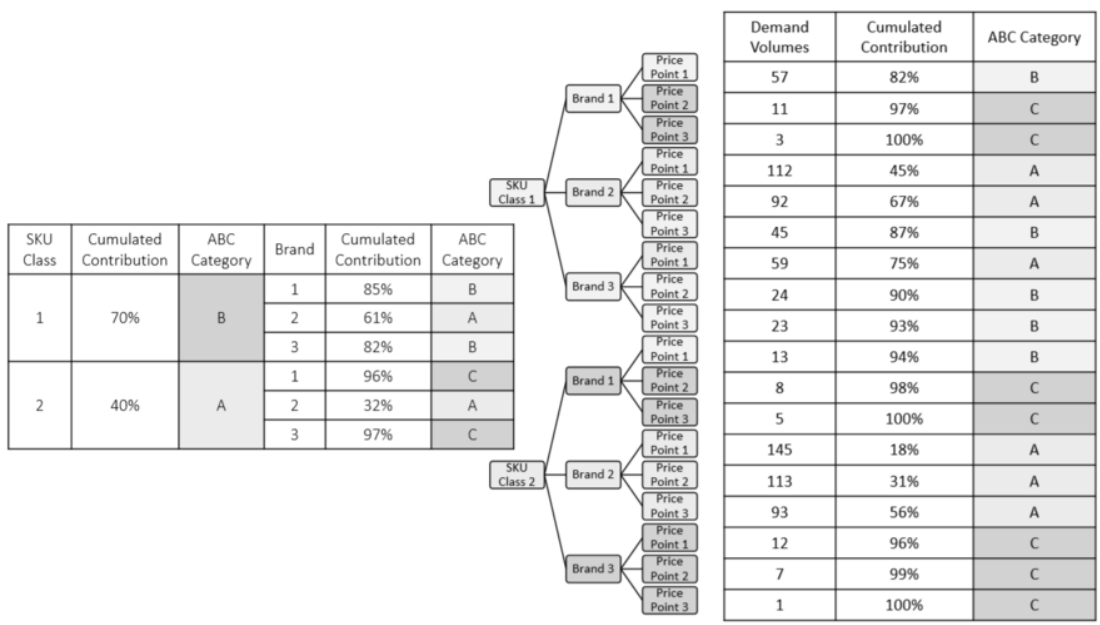

Figure 3. Assigning performance measures for SKU classes.

\subsection{Phase 2: TO-BE scenario}

\section{Step 2a: Identify possible SKU reduction initiatives}

Based on the insights from the previous step it is possible to generate different initiatives for reducing complexity costs by changing the product range (e.g., reducing the number of product variants, adjusting the product variants offered to each market, redesigning modules, changing product architecture, etc.) or by making changes in the business processes (e.g., reducing setup times, changing the order decoupling point and points of stock, changing delivery times, etc.) [11,17]. Furthermore, approaches such as product modularization [18] and design for manufacturing/assembly (DFA/DFM) [19] may be considered as a means to reduce product complexity. To identify possible initiatives for complexity reduction, interviews and/or focus groups should be held in which possible actions to reduce complexity are identified.

\section{Step 2b: Choose SKU reduction initiative}

To choose the best initiative for complexity reduction, each of identified initiatives should be compared and evaluated in terms of their cost profile. It can be assessed in terms of production, inventory, and freight costs, as well as its impact on delivery performance and quality. This will also reveal the potential savings (benefit) for each of the identified initiatives. In case it is necessary, the risk evaluation can be done in terms of the impact of this initiative on production and assembly processes.

\section{Research method}

Based on the literature, a framework is developed for analyzing and quantifying the product variety on different product levels and related to the process flow. The framework is tested in a company that offers hearing instruments, hearing implants, diagnostic instruments, and audio solutions. The company operates globally through a local presence in more than 30 countries and trusted distributors in more than 100 countries. In 2018 the company globally employed more than 14,000 persons. The case 
study company is a high-margin, sales-driven business that has doubled its revenue since 2010. This significant increase in sales resulted in the company's grow and development, increase in the number of employees and sales channels, and the purchase of three new brands. These circumstances directly resulted in an increase in the product variety offered to the market and growth of product, process, and organizational complexity. However, the complexity growth did not pay much attention to the executives until it began to impact operational performance. This was the main motivation for which this particular company was chosen to test the framework. To narrow the scope of the analysis and identify a good case study, the product families in the product portfolio are first analyzed in terms of SKU proliferation. Secondly, managers in the global supply chain are asked to select product families in which they experience decreased operational performance and to identify the processes in the supply chain, i.e., production, assembly, distribution, sales or aftersales in which this decreased operational performance occurs. Based on preliminary investigation, a newly launched product family of custom hearing aids is selected as a case study for testing the framework. The process identified for the study is local production of standard components and global assembly of final products.

The information needed is collected from analyzing bills of materials, performance measures, and lists of operations for the selected product families and by interviewing relevant stakeholders, such as production, warehouse, logistic managers, and product developers. The data were obtained from the company's PLM and ERP systems. The interviews included employees from the following departments: Global Production Planning (GPP), Global Custom Operations (GCO), Global Supply Chain (GSC), New Production Introduction (NPI) Production, Warehouse, Product Data Management (PDM). Information about the positions of the interviewees and the number of interviews in this study can be found in Table 2 .

Table 2. Questions guidelines and employees addressed for interviews

\begin{tabular}{|c|c|c|c|c|}
\hline Interviews topics & Department & Employee job position & $\begin{array}{c}\text { No. of } \\
\text { employees } \\
\text { interviewed }\end{array}$ & $\begin{array}{c}\text { No. of } \\
\text { interviews } \\
\text { with each }\end{array}$ \\
\hline \multirow{3}{*}{$\begin{array}{l}\text { Product variety and } \\
\text { performance }\end{array}$} & GCO & Director & 1 & 2 \\
\hline & NPI & $\begin{array}{l}\text { Group manager, Project } \\
\text { manager, NPI trainers }\end{array}$ & 4 & 12 \\
\hline & Production & Process eng. manager, Operator & 2 & 2 \\
\hline \multirow[t]{2}{*}{ Product information } & GPP & Demand planner & 1 & 2 \\
\hline & PDM & Head of tech. documentation & 1 & 10 \\
\hline Process information & Production & Process engineering manager & 1 & 3 \\
\hline \multirow{2}{*}{$\begin{array}{l}\text { Possible initiative for } \\
\text { complexity reduction }\end{array}$} & NPI & Group manager, Project & 4 & 1 \\
\hline & GSC & manager, NPI Trainers & 1 & 1 \\
\hline \multirow{2}{*}{$\begin{array}{l}\text { Process evaluation for } \\
\text { reduction of product } \\
\text { portfolio complexity }\end{array}$} & NPI & $\begin{array}{l}\text { Senior director, Group manager, } \\
\text { Project manager, NPI trainers, }\end{array}$ & 2 & 1 \\
\hline & GSC & Vice president, Director & 5 & 1 \\
\hline
\end{tabular}

\section{Case study}

The work carried out in the studied case is described below for each step of the suggested framework.

Step 1a: Define products and process scope

This complexity reduction project was initiated as the production and assembly experienced a significant decrease in performance measures, i.e. decreased delivery performance, increased supply chain costs, and increased component and finished goods 
inventories due to the introduction of new custom hearing aids. The focus group of employees from production, warehouse, logistic, and product development uniformly stated that the reason for this problem was the SKU proliferation of the new product family. However, none of the interviewed stakeholders were able to explain how the increased product variety of this product family impact performance measures and to provide transparency over this increased product variety. Therefore, it was decided to initiate a project to provide this transparency and show the impact of SKU complexity on performance measures. The product and process scope have been defined by the indicated focus group.

For the defined product and process scope, data were gathered for 830 product variants. Data included product data and demand volume for all 830 product variants, as well as naming convention and list of operations for given product family. The product data included a list of all SKUs for the identified product family that contained all product characteristics (components, size, color, etc.) and manufacturing Bill Of Materials (BOMs) for each product variant. Based on that it was possible to identify common components and number of variants for these components. The product data and naming convention for the given product family allows to define SKU characteristics (components, size, color). The process step mapping has been provided by production managers. To map the process at the high level, a production manager has been interviewed in iterative manner and asked what the order of identified SKU characteristics at different process steps is. This helped to identify how the complexity levels in the product variety arise at different process steps. Furthermore, the SKU characteristics have been distinguished to ones related to products and the ones related to customers. This led to the identification of SKU classes for product SKU characteristics and later visualize how customer SKU characteristics formed SKU for product variety.

\section{Step 1b: Identify and organize SKU characteristics}

As explained in the previous section, the SKU characteristics have been determined by product descriptions found in the product data. The explanation for different abbreviations has been found using naming convention for defined product family. The SKU characteristics were divided into characteristics related to product and customer SKU characteristics. The product SKU characteristics included: hearing aid audiological style, battery size, wireless link option, receiver size, push-button option, telecoil option, color, left/right side, and customer SKU characteristic included product brand, product model, and price point. The product SKU characteristics were classified, and $46 \mathrm{SKU}$ classes were defined to organize 830 product variants.

\section{Step 1c: Map product-process complexity}

Figure 4 illustrates how the defined SKU classes are mapped using the variant tree method. This technique allows users to clearly show how the complexity levels arise in the product assortment. However, it requires to be done in an iterative manner, as each of product SKU characteristics had to be properly linked to the process steps in the defined process flow. The steps in the process flow are mapped by using information from a list of operations. The product SKU characteristics are placed in the order accordingly to where in the production process, their corresponding product component is assembled. The variant tree with linked process steps is checked and validated by production managers and product developers. This allows to create a visual model that 
illustrates where and how the complexity levels in the product assortment arise in relation to the process flow.

\section{Step 1d: Identify complexity-performance trade-offs}

A traditional Pareto analysis was carried out to quantify the performance of product variants based on their demand volume. The low-runners and high-runners were identified using the traditional $80 / 20$ rule. The high-runners are product variants that contribute to $80 \%$ of sales, and the low-runners are product variants that contribute to the last $20 \%$ of sales. The analysis showed that high-runners count for 352 product variants, and low-runners count for 478 product variants. Once the performance for all product variants was known, the performance of the SKU classes is calculated using a weighted average for the performance of their corresponding product variants, where the weight factor is demand volume. The left side of Figure 4 illustrates the final results for performance calculations for SKU classes. Initially, 19 SKU classes are identified as potential candidates for reduction due to their low performance. Reduction of just $1 \mathrm{SKU}$ class may result in reduction of the 15-18 final SKU used for sales. Thus, there is great potential in this method for addressing SKU proliferation.

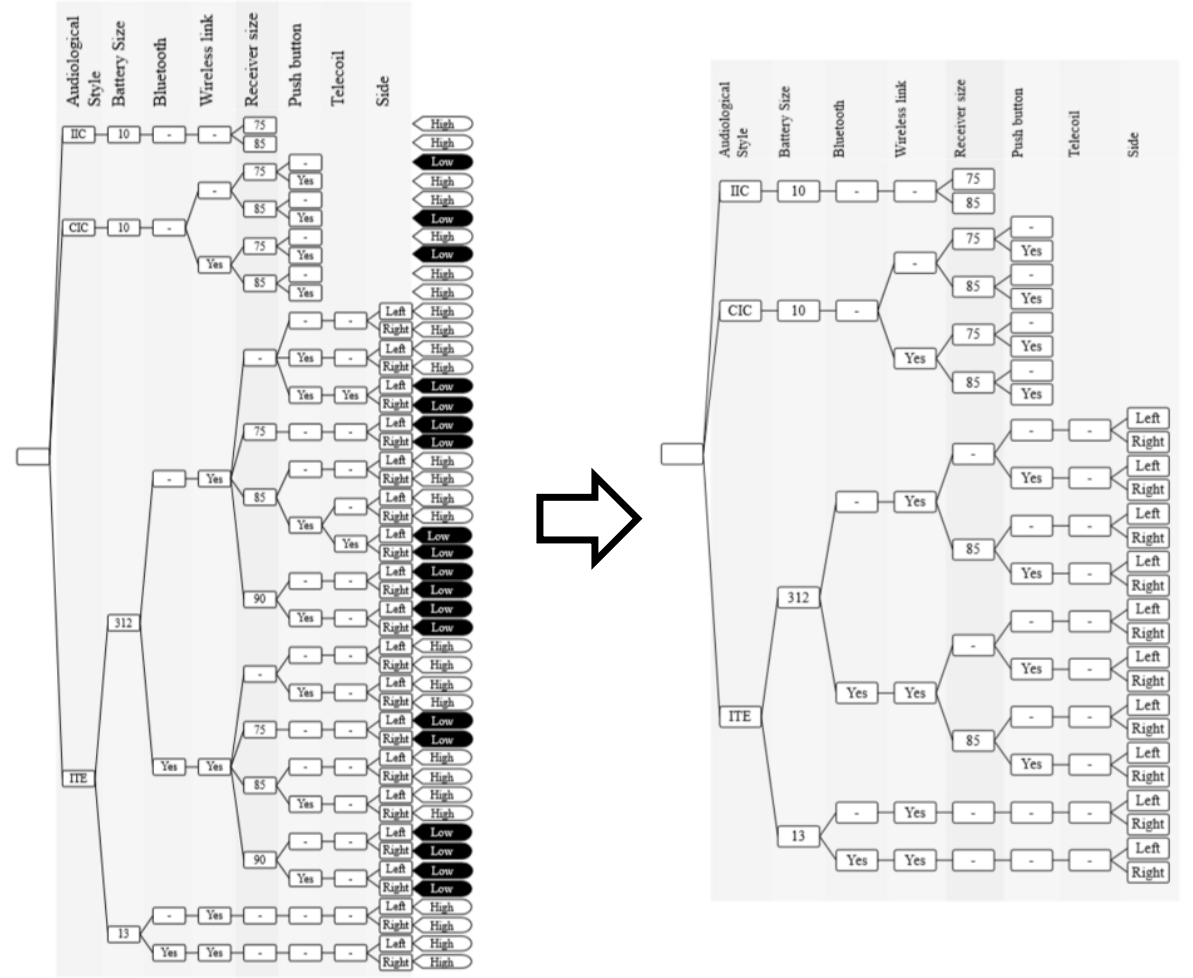

Figure 4. SKU classes performance based on demand volume of corresponding product variants.

\section{Step 2a: Identify possible SKU reduction initiatives}

The results were shared with the focus group and discussed. During the brainstorming session, stakeholders identified an initiative for reducing the number of SKU for given product family - namely to adjust order decoupling points for low performing SKU classes. The goal of this initiative was to centralize the production of real high-runners 
and decentralize the production of low runners. The product SKU characteristics for low performing SKU classes were analyzed. The conclusion was that in order to change decoupling points for low performing SKU classes, the assembly process for receiver and telecoil should be moved from standard production to custom production. Instead, the basic variants should be used in standard production and missing components to be assembled later in the process in custom production if a customer would place the order. The SKU reduction candidates were products with telecoil and receiver of size 75 and 90. However, it was not possible for all SKU classes as it could affect product quality.

\section{Step 2b: Choose SKU reduction initiative}

In the end, it was decided to discontinue 16 SKU classes, which resulted in a reduction of 290 unique SKUs. The right side of Figure 4 illustrates the SKU classes after reduction initiative. These account for $33 \%$ of product variety for this product family.

\section{Discussion and conclusion}

The literature provides means for reducing complexity based on analysis of the end product, but only to a limited extent attention is paid to understanding where this variance occurs in order fulfilment processes. To address this issue, this paper suggested and tested a framework for reducing product portfolio complexity based on process analysis.

Based on the literature, a simple six-step framework for SKU reduction was proposed. The first part of the framework concerns "AS-IS analysis", which includes the steps: (1a) Define product and process scopes of the project; (1b) Identify and organize SKU characteristics; (1c) Map product-process complexity; and (1d) Identify complexity-performance trade-offs. The second part of the framework concerns the "TOBE scenario", which includes the steps: (2a) Identify possible SKU reduction initiatives; and (2b) Choose SKU reduction initiative.

The application of the framework in a company case showed that complexity on different levels in the product structure is linked to the process flow. This analysis, therefore, provided a basis for the managers to make decisions on changing the decoupling points for low volume products, which in turn lead to a reduction of number of SKUs. The case data used for this analysis were easily available in the company's ERP and PLM system. The data pre-processing for this analysis required to clean product master data and add missing information from manufacturing BOMs, and further validation by product managers. This allowed to identify relevant SKU characteristics, identify SKU classes, and identify the number of final SKU for each of the class. On this basis, they mapped the arising levels of complexity in product structure by using the variant tree method. The production and assembly process mapping were done based on the interviews with production and warehouse managers. The production and assembly steps had to be selected for identified SKU characteristics. During the interviews, it occurred that mapping of product structure complexity had to be adjusted to process steps in the processes flow. Data analysis on final SKU included the traditional 80/20 Pareto analysis and calculation of the performance for identified SKU classes based on performance of final SKUs. Later the interview with product developers was held to and choose the initiative for complexity reduction and identify final candidates for SKU reduction. 
Some limitations to the findings need to be commented on. First, the framework was tested on a single case study, which obviously limits it generalizability. On the other hand, by collecting the product and performance data from multiple sources, the study provides a rich description of the application of the suggested approach. In this context, it should be noted that the case results were validated by the stakeholders and that the implementation resulted in SKU reduction of one product family by $33 \%$, without losing the variety offered to end customer. Thus, the findings lead to the recommendation on further research on different product types and/or different process steps in the supply chain.

In summary, the proposed framework allowed the case company to reduce internal product portfolio complexity without reducing product variety offered to the market. Future research needs to further test and develop the framework by applying it in other companies.

\section{References}

[1] T. Stabilen, M. Holweg and J. Miemczyk, Theoretical Versus Acutal Product Variety: How Much Customization do Cutomers Demand, International Journal of Operations \& Production Management, 31/3: 350-370, 2011.

[2] D.J. Closs, M.A. Jacobs, G.S. Webb, Toward a theory of competencies for the management of product complexity: six case studies, Journal of Operations management 26 (5), 590-610, 2008.

[3] B. Meeker, D. Parikh, M. Jhaveri, The complexity conundrum, Marketing Management 18 (1), 54-59, 2009.

[4] S. Berman, P. Korsten, Capitalizing on Complexity, Global Chief Executive Officer Study: 76. IBM Institute for Business Value, Somers, New York, 2010.

[5] R. Hoole, Drive complexity out of your supply chain. Harvard Business School Newsletter (January), 3-5, 2006.

[6] C.H. Fine, A.C. Hax, Manufacturing Strategy: A Methodology and an Illustration. Interfaces, 15/6: 28-46, 1985.

[7] A.T Kearney, Stop chasing your SKU tail, A.T. Kearney Executive Agenda, 2006.

[8] M.L. Fisher, C.D. Ittner, The impact of product variety on automobile assembly operations: empirical evidence and simulation analysis, Management Science 45 (6), 771-786, 1999.

[9] A. Trattner, et al., Product complexity and operational performance: A systematic literature review, NULL (2019), https://doi.org/10.1016/j.cirpj.2019.02.001

[10] Lancaster, K., 1990, The Economics of Product Variety: A Survey. Marketing Science, 9/3: 189-206.

[11] M.A. Jacobs, M. Swink, Product Portfolio Architectural Complexity and Operational Performance: Incorporating the Roles of Learning and Fixed Assets. Journal of Operations Management, 29:677-691, 2001.

[12] E.A. Silver, D.F. Pyke, R. Peterson, Inventory management and production planning and scheduling, 4th Edition, John Wiley and Sons, New York, 1998.

[13] D. Mahler, A. Bahulkar, Smart complexity, Strategy \& Leadership, Vol. 37 No. 5, pp. 5-11, 2009.

[14] T. van Kampen, R. Akkerman, D. Pieter van Donk, SKU classification: a literature review and conceptual framework. International Journal of Operations \& Production Management, 32 (7), 850-876.

[15] V. Krishnan, Karl T. Ulrich, Product Development Decisions: A Review of the Literature. Management Science, vol. 47, No. 1, 1-21, 2001.

[16] A. Lee Trattner, Product Variety Management in Process Industry Companies, Department of Management Engineering, Technical University of Denmark, Kongens Lyngby, 2019.

[17] J. Wikner and M. Rundberg, Integrating production and engineering perspectives on the customer order decoupling point, International Journal of Operations \& Production Management, vol. 25, No.1, pp. 623$641,2005$.

[18] K. Ulrich, Fundamentals of Product Modularity. In: Dasu S., Eastman C. (eds) Management of Design. Springer, Dordrecht., 1994

[19] Boothroyd, G. Design for assembly-The key to design for manufacture. Int J Adv Manuf Technol 2, 311 (1987). https://doi.org/10.1007/BF02601481

[20] Cavalieri S. et.al., A decision-making framework for managing maintenance spare part, Production Planning \& Control, 19(4), 379 - 396, 2008 